\title{
Orbital inflammatory disease in a child caused by a ruptured dermoid cyst misdiagnosed as orbital cellulitis
}

\author{
Hayrunnisa BEKIS BOZKURT ${ }^{1}$ D, Hulya GOKMEN SOYSAL ${ }^{2}$ \\ ${ }^{1}$ Department of Ophthalmology, Faculty of Medicine, Kafkas University, Kars, Turkey. \\ ${ }^{2}$ Department of Pediatrics, Faculty of Medicine, Kafkas University, Kars, Turkey.
}

Corresponding Author: Hayrunnisa BEKIS BOZKURT

E-mail: hayrunisabekis@hotmail.com

Submitted: 23.12.2020 Accepted: 12.03.2021

\begin{abstract}
One of the most common orbital tumors in childhood is orbital dermoid cysts. Cyst rupture or leakage of the cyst content is one of the rare causes of orbital inflammations and needs to be caught early. A five and a half year old boy was referred to us with the diagnosis of orbital cellulitis resistant to antibiotic therapy. Regarding his detailed medical history, physical examination and laboratory tests findings, he was diagnosed as orbital inflammatory disease caused by dermoid cyst rupture. He underwent surgical excision of the cyst after the inflammation was resolved with a short course of anti-inflammatory treatment. Although, it is rare, dermoid cyst rupture is one of the important causes of orbital inflammatory diseases and it must be kept in mind in the differential diagnosis of orbital infections. A detailed history and a careful examination helped us to make the correct diagnosis and avoided diagnostic delay and unnecessary treatment.

Keywords: Inflammation, Dermoid cyst, Child, Orbital cellulitis
\end{abstract}

\section{INTRODUCTION}

When we look at the etiology of orbital inflammatory disease (OID), we see a wide range of specific disease diagnoses to nonspecific inflammation [1]. The most important steps in the management of OID are to identify the etiology and select an appropriate treatment. From idiopathic inflammatory disease to systemic or local inflammatory conditions, to other related conditions such as trauma, infection, neoplasm or congenital malformation should be remembered in the differential diagnosis of OID [1,2].

Dermoid cysts constitute $5 \%$ of all orbital lesions in childhood [3]. These lesions are assumed to originate from ectodermal tissue deviating from sequencing that occurs when suture lines are closed during embryonic development $[4,5]$. Cyst rupture or leakage of the cyst content is one of the rare causes of orbital inflammation. Dermal appendages such as sebaceous material, oil, hair, and cholesterol crystals can mount as an intense inflammatory response in the surrounding tissue. We herein, present a child who had recurrent orbital inflammation caused by dermoid cyst rupture, initially misdiagnosed as orbital cellulitis.

\section{CASE REPORT}

A five and a half year old boy was consulted to our ophthalmology department for evaluation of right eyelid and periorbital swelling. He had been referred to pediatrics clinic from another medical center with the diagnosis of orbital cellulitis with resistant to antibiotic therapy.

His medical history before the admission of our center was obtained from medical records; he has been suffering from transient right eyelid swelling, each episode lasting about 1-2 weeks, 4-5 times over the last year. He had been admitted to another medical center a week ago for the same complaints. The laboratory and radiologic investigation results were obtained from medical records; haemogram, ESR, CRP was normal. Orbital computed tomography (CT) scan showed edematous and inflammatory thickening of the right eyelids and periorbital area. There was no evidence of sinusitis on CT. Intravenous medical therapy (Ceftriaxon $100 \mathrm{mg} / \mathrm{kg}$, Metronidazole $30 \mathrm{mg} /$ $\mathrm{kg}$ per day) was given for 5 days, with the diagnosis of orbital cellulitis. Then he was referred to our center because of no improvement.

How to cite this article: Bozkurt HB, Gokmen Soysal H. Orbital inflammatory disease in a child caused by a ruptured dermoid cyst misdiagnosed as orbital cellulitis. Marmara Med J 2021; 34: 348-350, doi: 10.5472/marumj.1013321 
On admission to our center, some diagnostic tests were done for differential diagnosis; haemogram and blood chemistry were in normal limits. Cultures for infection, $\mathrm{Clq}$ esterase inhibitor level for hereditary angioeudema, IgE level for allergic disease were evaluated but all results were normal ( $\operatorname{IgE}: 6,2 \mathrm{IU} / \mathrm{ml}, \mathrm{Clq}$ est inh:31 mg/dL).

On ophthalmic examination of the right eye, severe periorbital edema was evident that makes the eyelids difficult to open. There was no local temperature rise. There was neither conjunctival hyperemia nor ocular motility restriction. A subcutaneous, semi-mobile, hard mass about $1 \mathrm{~cm}$ diameter was palpated on the right lateral orbital area around the frontozygomatic suture. Visual acuity measurement and posterior segment examination were not possible. On examination of the left eye, visual acuity was 20/20 and there were no abnormal findings.

There was no history of trauma or systemic illness. A new CT scan was not needed because no evidence other than inflammation was seen in the previous CT. The preoperative CT findings show, irregular borders of ruptured dermoid cyst and edema, and it appears to be completely recovered after the operation on the postoperative CT (Figure 1a, Figure 1b).

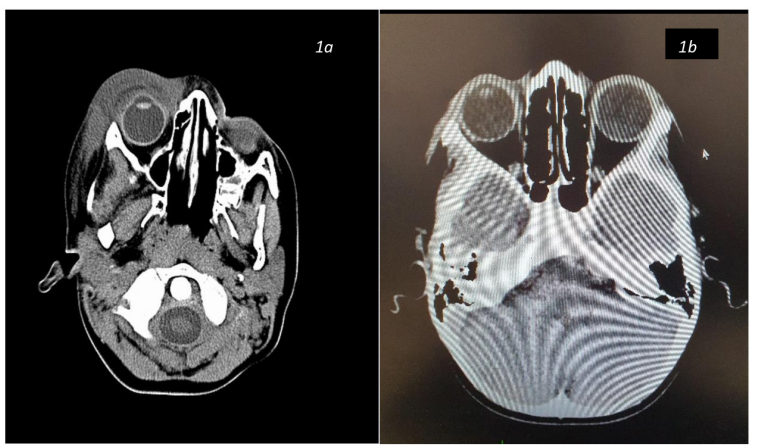

Figure 1a. Preoperative CT, dense inflammation and thickening of the eyelid and periorbital area on CT.

Figure 1b. Postoperative CT
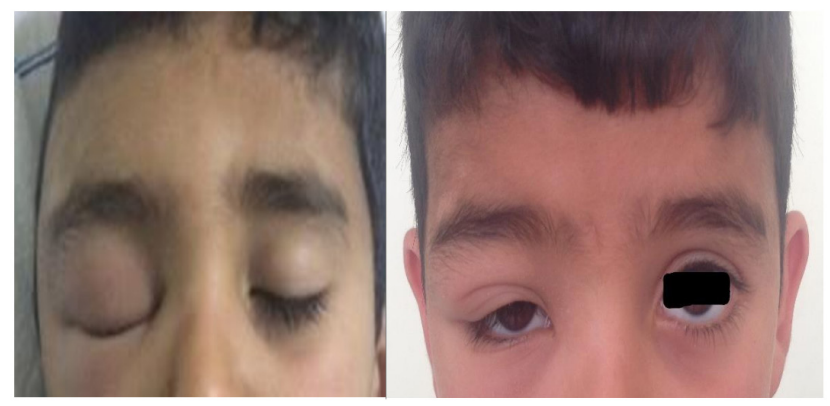

Figure 2. Appearance of patient (before and after) the anti-inflammatory therapy
Based on the history of recurrent periorbital swelling, spontaneous resolution and clinical findings, the patient was diagnosed as orbital inflammation related to ruptured dermoid cyst and medical treatment was rearranged. Nonsteroid antinflammatory agent (Ibuprofen $10 \mathrm{mg} / \mathrm{kg}$.day orally) was started while antibiotic treatment was chased. The symptoms and signs started to resolve rapidly beginning from the first day of the anti-inflammatory therapy (Figure 2a, Figure 2b). On orbital magnetic resonance imaging (MRI) obtained after the inflammation was resolved to a large scale there was a mild thickening and condensation around the lateral orbital area, interpreted as a cyst wall. Then the patient underwent surgery to remove all the cystic components. A lid crease incision was used to approach the lesion and cyst wall and other parts of lesion removed totally (Figure 3). Histopathological examination of the excision material was revealed the cyst wall and inflammatory and fibrous alteration surrounding the wall. After the first week of the surgery, the patient was nearly normal. In our 6-month follow-up, we could not detect recurrence in our patient.

Written consent was obtained from the patient's parents for the publication of this case report and any accompanying images.

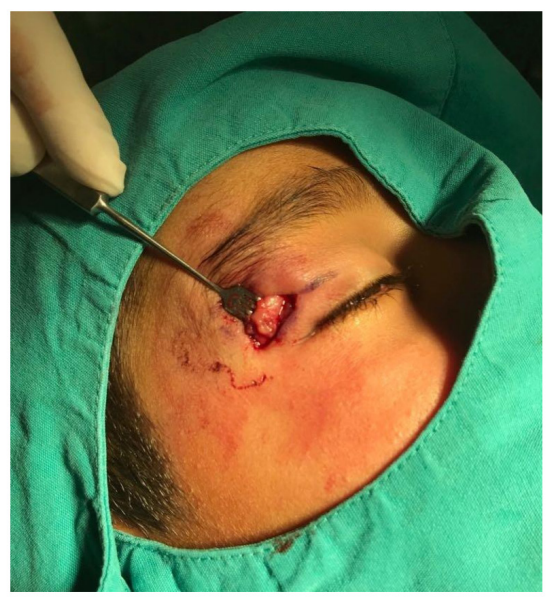

Figure 3. Intraoperative appearance of the cyst wall.

\section{DISCUSSION}

Orbital inflammation is due to the immune system's response, and the underlying cause is a clinical condition that needs clarification. It is important to investigate the etiology of autoimmune, structural, infectious, neoplastic and idiopathic causes. The clinician should carefully review all causes $[1,6]$.

The clinician needs to look over five main groups of diseases in differential diagnosis of orbital inflammation;

1) Systemic diseases are the most common cause, including thyroid orbitopathy, Wegener granulomatosis, sarcoidosis, and other connective tissue diseases like systemic lupus erythematosis, some hematologic diseaseS and vasculitis [1,7].

2) Infections, especially orbital cellulitis, are life-threatening complications and require early diagnosis and treatment. In any 
doubt, patients should be hospitalized for parenteral treatment as soon as possible and empirical intensive-broad spectrum antimicrobial therapy should be initiated without waiting for culture results [1]. In orbital infections, symptoms such as fever, acute phase reactant elevation and leukocytosis are encountered, whereas these are rare in non-communicable OID $[8,9]$.

But in our case, serological and orbital findings did not support the diagnosis of orbital infection.

3) Neoplastic diseases include lymphoproliferative disease, orbital metastasis, and primary malignant tumors such as rhabdomyosarcoma. Ruptured dermoid cysts can be classified in this group.

4) Vascular etiologies such as carotico-cavernous fistula or veno-lymphatic malformations also cause orbital inflammation.

5) Trauma and foreign body must be questionized in all OID patients.

In our patient's history, the intermittent nature and spontaneous resolution of the disease was remarkable. No evidence about systemic diseases was obtained from detailed medical and family history. The most important finding leading to our diagnosis was a palpable hard mass on lateral orbital area, suspicious of the cyst wall. Indeed, this has been confirmed by the way of surgery and histopathological examination of excised material.

Dermoid cysts are one of the rare but important causes of inflammation that are mostly seen in childhood. Depending on the location, it may vary clinically. There is a case of deeply located dermoid cyst in a 3-month-old baby reported from Nigeria in the literature [10]. The more superficially located ones can be overlooked until adulthood with false diagnoses such as cellulite. He was initially misdiagnosed with repeated hospital applications.

In a retrospective multicentric study, cases from 3 oculoplastic centers (Queen Victoria Hospital, East Grinstead, United Kingdom; King Abdulaziz University Hospital, Riyadh, Saudi Arabia; South Australian Institute of Ophthalmology and Visual Sciences, Australia) over a 10-year period, 29 of the total 86 cases were ruptured and only 2 were reported as spontaneous rupture. In the same study, it was pointed out that persistent inflammation was more pronounced after spontaneous rupture[11]. Our case was compatible with inflammation by ruptured dermoid cyst condition, in which infection and other causes are eliminated.

Dermoid cysts are defined as benign neoplasms and can cause complications such as spontaneous rupture and inflammation when not surgically removed. In a large ophthalmology clinic with 7 years of experience in Italy, 30 cases in the child age group were examined, by CT and / or MRI or echography which were suitable for diagnosis, and the best treatment was total excision, not biopsy. No recurrence has been reported after total removal [12]. We performed total excision due to the fact that our patient was a child and in the light of the literature. In our 6-month follow-up, we could not detect recurrence in our patient.

In conclusion, although it is rare, dermoid cyst rupture is one of the important causes of OID. A detailed history and a careful examination helped us to make the correct diagnosis and avoided diagnostic delay and unnecessary treatment.

Conflict of interest: The authors have no conflicts of interest to declare.

Funding: No financial support was received for this study..

Authors' Contributions: HG.: Idea/Consept. HBB.: Design. HG.:Control/Supervision. HBB.: Data collection. HG.: Literature review and writing. HBB.: Critical review. HBB.: References. reviewed and revised the manuscript. Both authors read and approved the final version of the article.

\section{REFERENCES}

[1] Belanger C, Zhang KS, Reddy AK, Yen MT, Yen KG. Inflammatory disorders of the orbit in childhood: a case series. Am J Ophthalmol 2010; 150:460-3. doi:10.1016/j. ajo.2010.05.012

[2] Salchow DJ. Orbital disorders in childhood. Klin Monbl Augenheilkd 2019;236:1351-71. doi: 10.1055/a-0972-4774.

[3] Reissis D, Pfaff MJ, Patel A, Steinbacher DM. Craniofacial dermoid cysts: histological analysis and inter-site comparison. Yale J Biol Med 2014; 87: 349-57.

[4] Siah WF, Al-Muhaylib AA, Rajak S, Selva D, Malhotra R, Alsuhaibani AH. clinical outcomes of ruptured periorbital and orbital dermoid cysts. Ophthal Plast Reconstr Surg 2017; 33: $264-67$

[5] Lenci LT, Shams P, Shriver EM, Allen RC. Dermoid cysts: clinical predictors of complex lesions and surgical complications. JAAPOS. 2017; 21: 44-7.

[6] Mombaerts I, Rose GE, Garrity JA. Orbital inflammation: Biopsy first. Surv Ophthalmol 2016; 61: 664-69.

[7] Cruz AAV. Orbital inflammation and infection versus neoplasia. In: Karcioglu ZA, ed. Orbital Tumors: Diagnosis and Treatment. New York: Springer, 2005: 317-28.

[8] Mouriaux F, Coffin-Pichonnet S, Robert PY, Abad S, MartinSilva N Orbital inflammation. J Fr Orbital 2014; 37:818-24.

[9] Williams KJ, Allen RC. Paediatric orbital and periorbital infections. Curr Opin Ophthalmol 2019 ;30:349-355.

[10] Fasina O, Ogun OG. Giant deep orbital dermoid cyst presenting early in infancy in a Nigerian child: a case report and review of the literature. J Med Case Reports 2012; 6: 320. doi:10.1186/1752-1947-6-320

[11] Siah WF, Al-Muhaylib AA, Rajak S. et al. Clinical outcomes of ruptured periorbital and orbital dermoid cysts. Ophthalmic Plastic \& Reconstructive Surgery 2017; 33: 264-7. doi: 10.1097/ IOP.000.000.0000000735

[12] Cavazza S, Laffi GL, Lodi L, Gasparrini E, Tassinari G. Orbital dermoid cyst of childhood: clinical pathologic findings, classification and management. Int Ophthalmol 2011;31:93-7. doi: 10.1007/s10792.011.9419-y 